\title{
The Effect of a Two-hour, Room Temperature Incubation of Human Spermatozoa in TEST-Yolk Buffer on the Rate of Fertilization in Vitro
}

\author{
Julie R. Jensen, ${ }^{1}$ John H. Walker, ${ }^{1}$ Amin A. Milki, ${ }^{2}$ Lynn Westphal, ${ }^{2}$ and Barry Behr ${ }^{2,3}$
}

Submitted December 31, 2003; accepted March 22, 2004

Purpose: To reassess the use of TEST-yolk buffer (TYB) in an in vitro fertilization (IVF) program by comparing fertilization rates achieved in a glucose-free cleavage medium by the standard IVF preparation of sperm versus a 2 -h, room temperature incubation of sperm in TYB.

Methods: Oocytes collected for IVF were randomly split into two groups and inseminated with either TYB-treated sperm or IVF-prepared sperm.

Setting: Stanford Reproductive Endocrinology and Infertility Center.

Patients: Fifty couples undergoing IVF with at least 10 mature oocytes. Main Outcome Measures: Fertilization rates in vitro.

Results: Fertilization rates were significantly higher $(p=0.015)$ with TYB treatment. The average $2 \mathrm{PN}$ fertilization rate was $49.6 \%(188 / 379)$ for the IVF group and $57.4 \%(221 / 385)$ in the IVF with TYB group.

Conclusions: A 2-h, room temperature incubation of sperm in TYB produces significantly higher $2 \mathrm{PN}$ fertilization rates as compared to standard IVF preparation of sperm in a current generation cleavage medium.

KEY WORDS: Fertilization; TEST-yolk buffer.

\section{INTRODUCTION}

The value of TEST-yolk buffer (TYB) in cryopreservation is well documented and widely accepted. Additional benefits of TYB have been explored due to the possibility that TYB is capable of enhancing the properties of fresh semen as well. The use of TYB has been shown to increase the binding capacity of spermatozoa in the hemizona assay (HZA) (1-4) and to increase penetration in the hamster egg penetration

\footnotetext{
${ }^{1}$ California Polytechnic State University, San Luis Obispo, California.

${ }^{2}$ Department of Obstetrics and Gynecology, Stanford University School of Medicine, 300 Pasteur Drive, Stanford, California 94305-5317.

${ }^{3}$ To whom correspondence should be addressed; e-mail: gjcarden@ stanford.edu.
}

assay (HEPA) (5-9). Enhanced fertilization has also been observed with intrauterine inseminations (IUI) and intraperitoneal inseminations (IPI). In other studies, TYB treated sperm showed benefit by producing pregnancies in patients with previous failures, achieving pregnancies resulting from only embryos originating from TYB treated sperm and also producing embryos with better morphology (10-12).

In preparing sperm samples for various assisted reproductive technology (ART) procedures, TYB treatment appears beneficial to fertilization and conception rates. Incubation in TYB may preserve the viability of sperm and aid in fertilization by interacting with the sperm membrane (13-15), synchronizing capacitation and the acrosome reaction $(2,7-9,16)$, and increasing the percentage of sperm capable of fertilizing an oocyte $(8,17)$. 
Although TYB is often applied overnight at temperatures of around $4{ }^{\circ} \mathrm{C}$, several studies have shown similar benefits from 2 -h, room temperature incubations of spermatozoa $(1,2,4)$ in bioassays. In addition, in a single limited study Barak et al. showed a benefit of short exposure to TYB for male factor patients (13). In contrast to the Barak study, this study was designed to evaluate the practical use of TYB in an IVF program on all scheduled IVF cycles by splitting patients' oocytes between the TYB and non TYB-prepared sperm, thereby allowing patients to serve as their own controls. This is important when using a nonnormalized patient population. Fertilization by either conventional IVF-prepared sperm (IVF) or 2-h, room temperature TYB-incubated sperm (IVF-T) was assessed as a means of determining the effect of TYB use.

\section{MATERIALS AND METHODS}

On the day of egg retrieval for IVF, consenting patients provided freshly ejaculated semen to the laboratory for processing. Following liquefaction, each sample was evenly split into two aliquots.

The IVF semen aliquot was layered over a density gradient column containing $1.0 \mathrm{~mL}$ Pure Sperm 40 over $1.0 \mathrm{~mL}$ Pure Sperm 80 (Nidacon International $\mathrm{AB}$, Gothenburg, Sweden). Centrifugation followed at $300 \times g$ for $20 \mathrm{~min}$. Supernatant and media were removed and the remaining sperm pellet was resuspended in $5.0 \mathrm{~mL}$ Quinn's Advantage Fertilization Medium (Sage Biopharma, Bedminster, NJ) supplemented with 5\% Human Serum Albumin (Sage Biopharma, Bedminster, NJ). The suspension was centrifuged for $10 \mathrm{~min}$ at $500 \times \mathrm{g}$, and the supernatant removed down to $0.5 \mathrm{~mL}$. Purified sperm were placed in a $37^{\circ} \mathrm{C}, 5 \% \mathrm{CO}_{2}$ incubator until insemination.

The IVF-T semen aliquot was diluted 1:1 withTYB Refrigeration Medium (Irvine Scientific, Santa Ana, $\mathrm{CA})$ and incubated in the dark for $2 \mathrm{~h}$ at room temperature. Centrifugation followed at $500 \times g$ for $10 \mathrm{~min}$. Supernatant and media were removed down to $1.0-2.0 \mathrm{~mL}$, with the remaining sperm and media layered over a density gradient column and processed as described above for the IVF portion.

Oocytes for each patient were randomly divided into two groups and placed in $150 \mu \mathrm{L}$ drops of equilibrated cleavage media (Sage Biopharma, Bedminster, NJ) with no more than five oocytes per drop. Insemination took place with approximately 100,000 motile sperm per $\mathrm{mL}$ for both the IVF or IVF-T
Table I. Postinsemination Results

\begin{tabular}{lcccc}
\hline & $2 \mathrm{PN}(\%)$ & $3 \mathrm{PN}(\%)$ & $1 \mathrm{PN}(\%)$ & Nonfert (\%) \\
\hline IVF prepared & 49.6 & 9.5 & 3.7 & 36.9 \\
IVF-T prepared & 57.4 & 9.1 & 4.9 & 28.3 \\
\hline
\end{tabular}

groups $2-4 \mathrm{~h}$ post egg retrieval. No accommodations were made for oocyte maturity, i.e. all oocytes were inseminated at the same time, regardless of their appearance with respect to maturity. Fertilization was assessed for each group 16-19 h postinsemination by the presence of pronuclei. Institutional review board approval was obtained for laboratory chart review.

\section{RESULTS}

Data were collected for 50 couples resulting in a total of 764 oocytes. Three hundred and eighty-five were allocated to the IVF-T group and 379 oocytes allocated to the IVF group. The average $2 \mathrm{PN}$ fertilization rate was $49.6 \%$ (188/379) for the IVF group and $57.4 \%(221 / 385)$ in the IVF-T group, $p=0.015$. Abnormal fertilizations were not different. These percentages are displayed in Table I.

Because the number of oocytes differed widely among the couples (min. 6, max. 31, avg. 15), the 2PN fertilization rate was modeled using a logistic regression, which treats each of the 764 oocytes as an individual observation.

Since the oocytes and sperm were split for each couple, each couple served as its own control. The 50 couples were treated as levels of a blocking factor to control for the variable fertilization rates from couple to couple. The fixed treatment factor had two levels, IVF and IVF-T.

After controlling for the couple effect, the analysis showed that the IVF-T treatment had significantly higher 2PN fertilization rate $(p$-value $=0.015)$ with an odds ratio of 1.47. This indicates that, on average, the odds of $2 \mathrm{PN}$ fertilization were $47 \%$ higher for the IVF-T treatment when compared to the IVF treatment. With $95 \%$ confidence, the odds of $2 \mathrm{PN}$ fertilization increased by somewhere between 8 and $102 \%$ (18).

Figure 1 shows the actual proportion of $2 \mathrm{PN}$ fertilizations per treatment-couple combination versus the estimated probability of $2 \mathrm{PN}$ fertilization from our model. Perfect model estimates would fall along the diagonal line the plot. It is worthwhile to note 


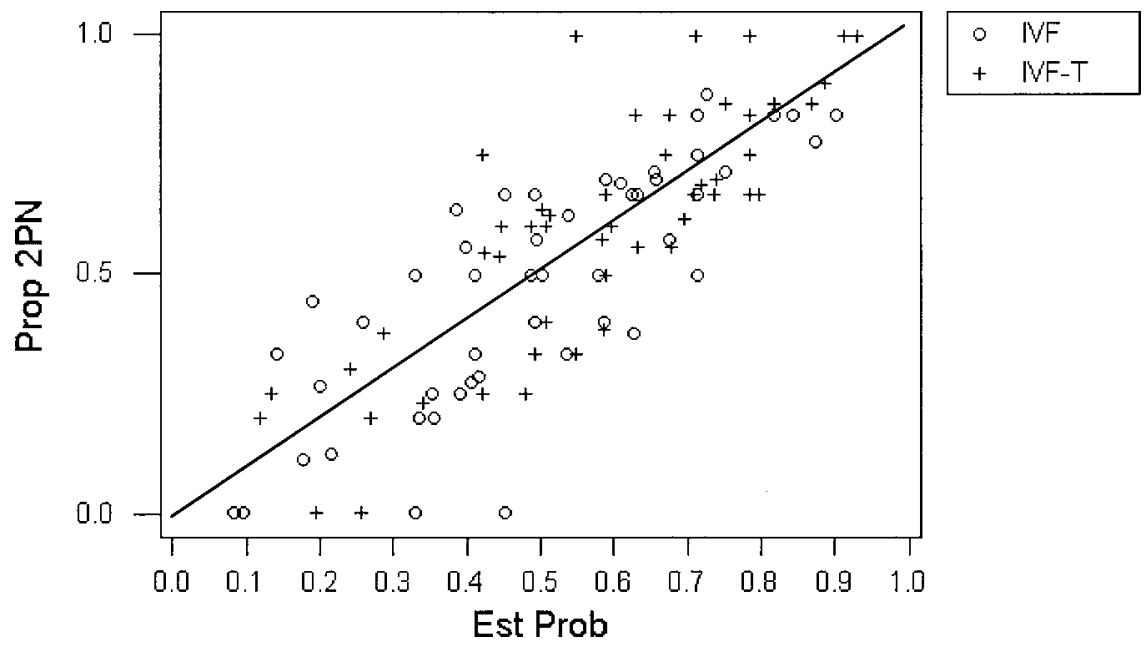

Fig. 1. Proportion of 2PNs vs. probability of fertilization.

that there are more "+" symbols indicating IVF-T treatment in the upper right hand corner with better fertilization and more "o" symbols indicating IVF treatment lower on the graph.

A Hosmer-Lemeshow test indicated that the model fit well ( $p$-value $=0.864$, low $p$-value indicates poor fit). In addition, a Receiver Operating Characteristic (ROC) curve analysis was performed. The resulting ROC curve, shown in Fig. 2, plots the probability of the model to successfully predict $2 \mathrm{PN}$ fertilization (sensitivity) versus the probability of misclassifying a negative result as $2 \mathrm{PN}(1$ - specificity) for 99 different discrimination levels. A diagonal line with area 0.5 below the curve would indicate that the model is no more successful than a coin toss at discriminating

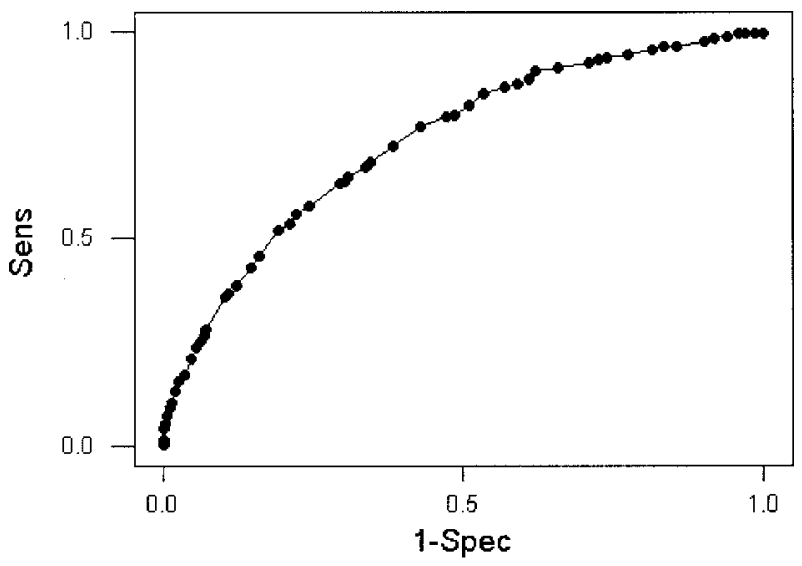

Fig. 2. ROC curve. between 2PN and the negative results. For our model, the area under the curve equals 0.739 , which indicates that the model discriminates reasonably well (18).

\section{DISCUSSION}

Although not routinely used in IVF, the potential benefits of TYB addition to semen have been demonstrated through previous studies. These studies have primarily been performed with overnight incubation at $4{ }^{\circ} \mathrm{C}$ for ART or, with shorter incubation times have demonstrated favorable outcomes in bioassays. Despite these findings, the mechanism of action is difficult to decipher. It does appear that TYB plays a role in preserving the integrity of sperm, synchronizing and inducing capacitation and the acrosome reaction, and improving binding to and penetration of the oocyte.

The environment of the spermatozoa may be improved by TYB preincubation, especially when a current generation glucose-free medium is used for sperm washing and insemination as there is still much debate about the sperms requirement for glucose in the fertilization media. The egg yolk proteins in TYB are capable of binding heavy metals, as well as stabilizing $\mathrm{pH}$ and $\mathrm{CO}_{2}$ levels in the media (19). These proteins may also help maintain the colloid pressure external to the sperm cell (14) and reduce free radical damage (13). In addition, the TES and Tris buffers in TYB potentially aid in the creation of a more physiologic environment in which fertilization can take place (8). 
Stabilization of the sperm membrane, as well as the internal structures of the sperm, is an additional way in which TYB potentially preserves sperm. The egg yolk proteins present in TYB conceivably preserve membrane integrity by the binding of entire lipoproteins to the sperm membrane (14). Some components of the egg yolk may in fact enter the sperm cell itself and produce some internal changes in stability that are necessary for continuing the fertilization process (13).

The capacitation of sperm is a critical step in the fertilization process. It has been suggested that the TES and Tris buffers in TYB are directly responsible for capacitation by removing proteins coating the sperm membrane, altering the fluidity of the membrane (16). Theoretically, the changes induced by these buffers are what allow capacitation and, subsequently, the acrosome reaction to take place (16). It has also been suggested that the removal of the sperm from TYB is what synchronizes capacitation and the acrosome reaction $(8,9)$. While sperm are suspended in TYB, the yolk lipids may allow capacitation to take place but prevent the actual acrosome reaction $(1,7)$. Once the sperm are washed free from the buffer, they are synchronized to undergo the acrosome reaction within the same time period $(1,7)$. It is also possible that TYB itself is responsible for triggering the acrosome reaction. The high-density lipoproteins in TYB potentially increase the permeability of the membrane to calcium by decreasing the cholesterol/phospholipid ratio in the sperm membrane, thereby initiating the acrosome reaction (16). TYB may also correct a defect in the acrosome reaction, increasing the percentage of sperm capable of undergoing the reaction (17). A more effective capacitation and/or a larger percentage of sperm becoming capacitated could play a role in the presence of a higher percentage of acrosome reacted sperm, which may in turn produce higher fertilization rates in vitro. One factor that potentially contributes to some failed or reduced fertilization in routine IVF may be the inability of sufficient normal sperm to reach the zona pellucida. It takes many sperm to "pave the way" for the fertilizing sperm to reach the zona. Semen samples that have a high percentage of sperm that either, spontaneously deploy the acrosome reaction or those who fail to acrosome react at all, may be the ones that benefit from TYB preincubation. Since these patients are not easily identified prior to the "the human sperm penetration assay" (IVF), the use of routine TYB preincubation for first time IVF patients could be beneficial.
Various studies describe the capacity of TYB to enhance the binding of sperm to the zona pellucida. It has been determined that TYB treatment increases the number of sperm capable of binding to the zona pellucida, as indicated by the fact that the TYB incubated sperm show higher binding results in the HZA than sperm prepared by other methods $(3,4)$. The suggestion has been made that the heat shock step present in most TYB treatment protocols is more important in increasing the fusogenic capabilities of sperm than the actual presence of TYB (8). However, in this study and other studies looking at bioassays, an increase in the ability of sperm to bind to the zona pellucida following a 2-h, room temperature incubation of sperm in TYB has been observed, suggesting that its effect is unrelated to the temperature shock experienced in the traditional TYP exposures $(1,4)$. In addition, the treatment of sperm from severely teratozoospermic samples in TYB for $2 \mathrm{~h}$ at room temperature has been found to increase the hemizona index (HZI) values in the HZA to almost normal threshold levels (2).

Although not analyzed in a routine IVF workup, the ability to increase the penetration rate of sperm is a property that has been attributed to TYB. Univeral improvement in the SPA and the HEPA has been observed with TYB treatment, regardless of duration or temperature of treatment (5-9). TYB has been used in various fertility treatments in an attempt to increase the fertilization and pregnancy rates. Couples attempting to become pregnant through IUI have realized greater success with TYB treated sperm, as compared to the standard swim-up preparation method (17). The fertilizing capacity of sperm in vitro is also enhanced by treatment with TYB, primarily accomplished with overnight incubation (11-13). It appears that, among couples who enjoy a high fertilization rate from an IVF procedure, TYB treatment does little to enhance the performance of the sperm. However, couples with a history of low fertilization may realize a significant increase in fertilization following TYB treatment (13). The maximum benefit of TYB addition in our study also appeared to be with the patients that had poor fertilization in the IVF group.

Although the exact way in which TYB acts to improve a sperm sample remains unclear, the benefits of its use are evident in this study. In particular, using patients as their own controls by splitting oocytes between the two treatment groups and using the appropriate statistics to analyze the outcomes, allowed us to effectively asses the true effects of the TYB and not patient variables. Incubation of sperm with TYB 
significantly improves the rate of $2 \mathrm{PN}$ fertilization and may decrease the incidence of unexplained nonfertilization in IVF. If routinely applied, TYB use may give clinics the opportunity to use less ICSI without compromising fertilization as is done in our center. Even though ICSI has not been conclusively linked to the host of problems claimed by its detractors, TYB use provides a viable alternative for cautious patients whose semen parameters do not totally preclude routine IVF. Whether applied on a case-by-case basis or to an entire population of IVF patients, TYB treatment shows great promise in enhancing fertilization.

\section{REFERENCES}

1. Gamzu R, Yavetz H, Lichtenberg D, Paz G, Homonnau ZT, Yogev L: The effect of egg yolk on the binding capacity of human spermatozoa to zona pellucida. Fertil Steril 1994;62:12211225

2. Gamzu R, Yogev L, Botchan A, Amit A, Lessing JB, Lichtenberg D, Paz G, Yavetz H: Effect of sperm preparation with TEST yolk buffer on sperm-binding capacity under hemizona assay conditions. Andrologia 1997;29:17-21

3. Lanzendorf SE, Holmgren WJ, Jeyendran RS: The effect of egg yolk medium on human sperm binding in the hemizona assay. Fertil Steril 1992;58:547-550

4. Yogev L, Homonnai ZT, Gamzu R, Amit A, Lessing JB, Paz $\mathrm{G}$, Yavetz $\mathrm{H}$ : The use of hemizona assay in the evaluation of the optimal sperm preparation technique. Hum Reprod 1995;10:851-854

5. Bolanos JR, Overstreet JW, Katz DF: Human sperm penetration of zona-free hamster eggs after storage of the semen for 48 hours at $2^{\circ} \mathrm{C}$ to $5^{\circ} \mathrm{C}$. Fertil Steril 1983;39:536-541

6. Chan SYW, Li SQ, Wang C: TEST-egg yolk buffer storage increases the capacity of human sperm to penetrate hamster eggs in vitro. Int J Androl 1987;10:517-524

7. Falk RM, Silverberg KM, Fetterolf PM, Kirchner FK, Rogers BJ: Establishment of TEST-yolk buffer enhanced sperm penetration assay limits for fertile males. Fertil Steril 1990;54:121-126
8. Jacobs BR, Caulfield J, Boldt J: Analysis of TEST (TES and Tris) yolk buffer effects on human sperm. Fertil Steril 1995;63:1064-1070

9. Scobey MJ, Biefield P, Gunawardana VK, Jeyendran RS: Influence of media and thermal shock on sperm capacitation. Andrologia 1996;28:171-174

10. Ragni G, Lombroso Finzi GC, Caccamo A, Dalla Serra A, Crosignani PG: Enhanced quality of capacitated spermatozoa from oligoasthenozoospermic men after incubation in test yolk. medium. Andrologia 1993;25:337-339

11. Paulson RJ, Sauer MV, Francis MM, Macaso TM, Lobo RA: A prospective controlled evaluation of TEST-yolk buffer in the preparation of sperm for human in vitro fertilization in suspected cases of male infertility. Fertil Steril 1992;58:551-555

12. Katayama KP, Stehlik E, Roesler M, Jeyendran RS, Holmgren WJ, Zaneveld LJD: Treatment of human spermatozoa with an egg yolk medium can enhance the outcome of in vitro fertilization. Fertil Steril 1989;52:1077-1079

13. Barak Y, Amit A, Lessing JB, Paz G, Homonnai ZT, Yogev L: Improved fertilization rate in an in vitro fertilization program by egg yolk-treated sperm. Fertil Steril 1992;58:197-198

14. Foulkes JA: The separation of lipoprotein from egg-yolk and their effect on the motility and the integrity of bovine spermatozoa. J Reprod Fertil 1977;49:277-284

15. Zavos PM, Goodpasture JC, Zaneveld LJD, Cohen MR: Motility and enzyme activity of human spermatozoa stored for 24 hours at $+5^{\circ} \mathrm{C}$ and $-196^{\circ} \mathrm{C}$. Fertil Steril 1980;34:607609

16. Ijaz A, Hunter AG, Graham EF: Identification of the capacitating agent for bovine sperm in egg yolk-TEST semen extender. J Dairy Sci 1989;72:2700-2706

17. Ragni G, De Laurentis L, Ambrogio GD, Pellegrini S, Maggioni P, Vegetti W, Perotti L, Dalla Serra A, Parazzini F, Crosignani PG: Semen preparation by standard swim-up versus swim-up with test yolk buffer incubation in intrauterine insemination: A randomized study. Hum Reprod 1998;13:18591863

18. Hosmer DW, Lemeshow S: Applied Logistic Regression, 2nd edn. New York, Wiley, 2001, 672 pp

19. Zavos PM, Correa JR, Zarmakoupis-Zavos PN: Antisperm antibody treatment mode: Levels of antisperm antibodies after incubation with TEST-yolk buffer and filtration using the SpermPrep ${ }^{\text {TM }}$ II method. Fertil Steril 1998;69:517-521 